\title{
Addition of dexmedetomidine to QX-314 enhances the duration of cutaneous analgesia in rats
}

\author{
Zhao W. L. ${ }^{1}$, Shi Y. ${ }^{1}$, Liu J. ${ }^{1}$, Jiang J. L. ${ }^{2}$, Zhang W. S. ${ }^{3}$ \\ ${ }^{1}$ Sichuan University, Dept of Anaesthesiology, Chengdu, China \\ 2Zun Yi Medical University, Dept of Anaesthesiology, Zun Yi, China \\ ${ }^{3}$ West China Hospital of Sichuan University, Dept of Anaesthesiology, Chengdu, \\ China
}

\begin{abstract}
Background and Goal of Study:
Previous studies have demonstrated that the combination of dexmedetomidine with commonly-used local anesthetics, such as ropivacaine and bupivacaine, can improve the duration of anaesthesia. We designed this study to observe whether adding dexmedetomidine to QX-314, the quaternary lidocaine derivative, would enhance the onset and duration of cutaneous blockade in rats in order to produce longacting analgesia.
\end{abstract}

\section{Materials and Methods:}

The study was approved by the Institutional Animal Experimental Ethics Committee of Sichuan University with the approval number: 2015014A. Fifty-six Sprague-Dawley rats ( $\mathrm{n}=8$ /group, 230-275 g) were divided into seven groups, $35 \mathrm{mM}$ QX-314, dexmedetomidine $(5.3 \mu \mathrm{M}, 26.4 \mu \mathrm{M}, 52.8 \mu \mathrm{M})$ with or without $35 \mathrm{mM}$ QX-314. The rats' dorsal surface of the fixed thoracolumbar area were shaved. The tested solutions were injected by using a 26-guage needle subcutaneously. Cutaneous analgesia was defined as the blockade of the cutaneous trunci muscle reflex. Six pinpricks per test were enough to obtain the results and the absence of lower or equal to three of six responses were defined as complete cutaneous blockade. The assessment was performed at the intervals after injection as follows: $15 \mathrm{~min}, 30 \mathrm{~min}, 1$ h, $2 \mathrm{~h}, 3 \mathrm{~h}, 4 \mathrm{~h}, 6 \mathrm{~h}, 8 \mathrm{~h}, 10 \mathrm{~h}$ until the values were up to the baseline (six responses per test).The data were presented as medians with interquartile ranges. The Mann-Whitney $U$ test with Bonferroni's correction $(\alpha=0.05 / 6)$ was used for multiple comparisons of duration of complete block.

\section{Results and Discussion:}

Duration of complete cutaneous blockades were enhanced when 35 $\mathrm{mM}$ QX-314 plus dexmedetomidine $(5.3 \mu \mathrm{M}, 26.4 \mu \mathrm{M}, 52.8 \mu \mathrm{M})$ compared with QX-314 alone (Fig. 1, P $<0.0083$ ). However, there were no statistic difference among three groups of dexmedetomidine (P > 0.0083). In addition, concentrations of $5.3 \mu \mathrm{M}, 26.4 \mu \mathrm{M}$ and 52.8 $\mu \mathrm{M}$ dexmedetomidine exhibited no effective blockade.

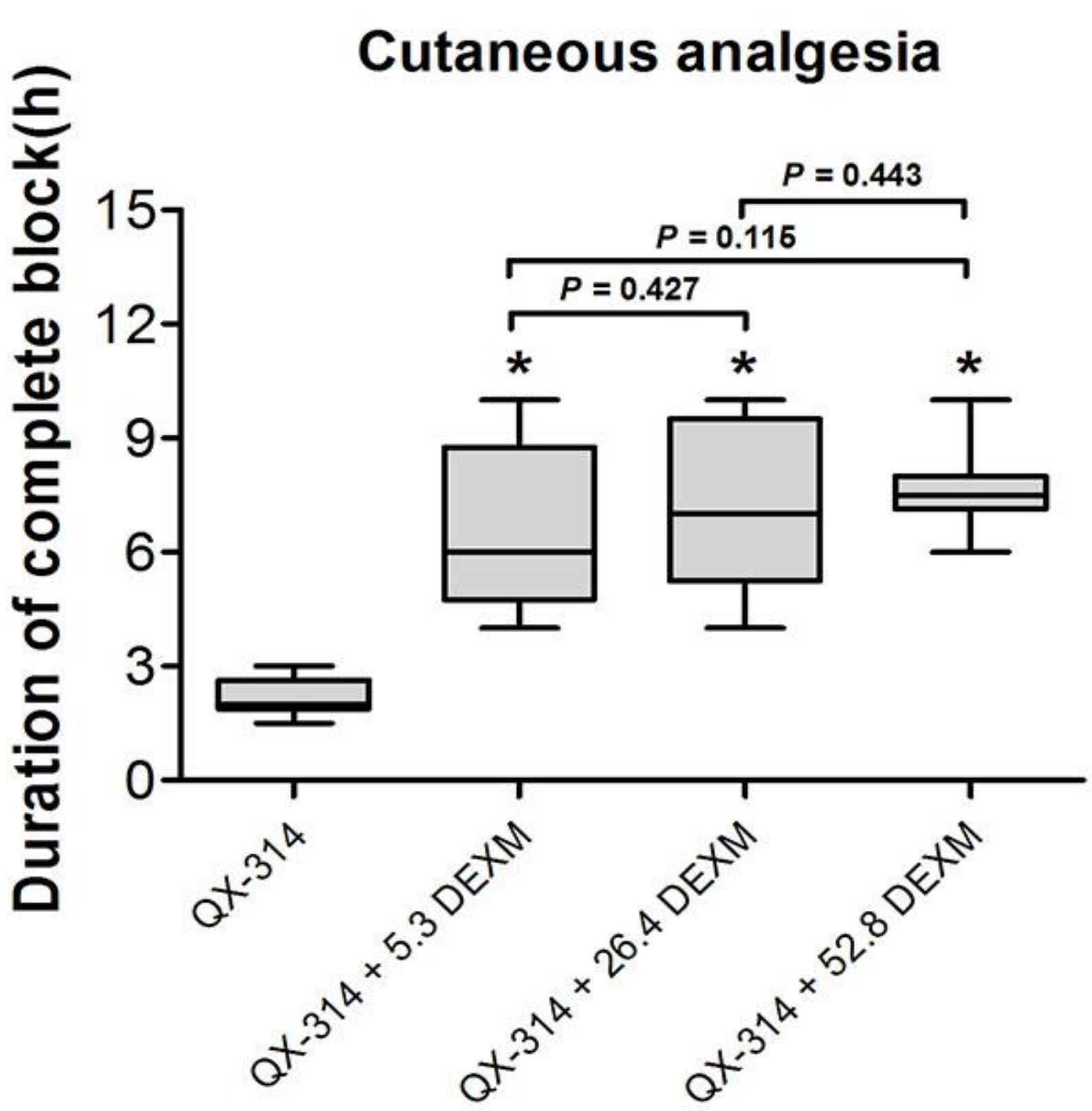

Figure 1. Duration of complete cutaneous block between $35 \mathrm{mM}$ QX-314 with or without dexmedetomidine at different concentrations ( $\mathrm{n}=8$ /group). Dexmedetomidine added to QX-314 increased the duration of complete cutaneous blockade when compared with QX314 alone ( $\mathrm{P}<0.0083)$, but there were not in a dose-dependent fashion when compared among groups of three concentrations of dexmedetomidine $(\mathrm{P}>0.0083$ and detailed $\mathrm{P}$ values noted in the figure). The center line is the median, the lower and upper boundaries are the 25th and 75th percentiles, and the error bars are the minimum and the maximum. $* \mathrm{P}<0.05 / 6$ (0.0083). $(\mathrm{DEXM}=$ dexmedetomidine)

\section{Conclusion:}

In summary, this animal study verified the hypothesis that coadministration of QX-314 with clinical-dose dexmedetomidine produced a synergistic anaesthetic effect to enhance the duration of cutaneous block in rats. Accordingly, dexmedetomidine may be a good adjuvant to QX-314 for long-acting anaesthesia. Further study is needed to testify other methods of local anaesthesia and elucidate the specific mechanism.

Conflicts of interest: The authors have no conflicts of interest.

Funding: Supported by the grant 2014ZX09101001-003 from the National Science and Technology Major Project of China, Beijing, P.R. China.

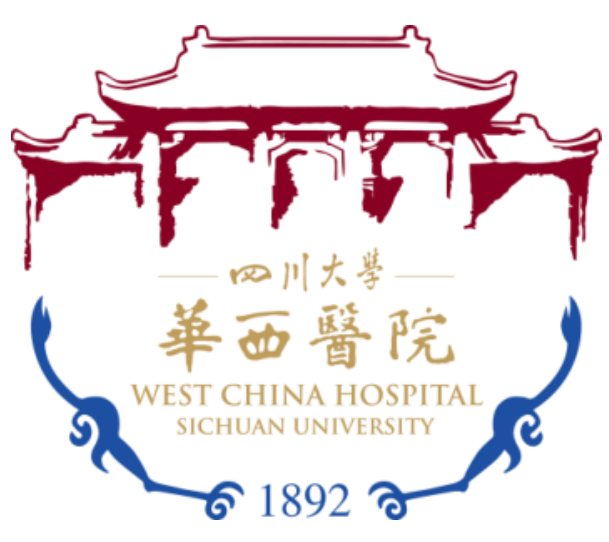

